
\title{
$\begin{array}{ll}\text { Research Square } & \begin{array}{l}\text { Preprints are preliminary reports that have not undergone peer review. } \\ \text { They should not be considered conclusive, used to inform clinical practice, } \\ \text { or referenced by the media as validated information. }\end{array}\end{array}$
}

\section{PELP1 expressed in non-small cell lung cancer and promotes tumor cell proliferation, metastasis, and drug resistance through MAPK signaling pathway.}

Xingen Wang

1.Southern medical university;2.Peking University Shenzhen Hospital

Weihua Ying

Peking University Shenzhen Hospital

Li Liang ( $\nabla 6686670 @ q q . c o m)$

southern medical university https://orcid.org/0000-0001-5918-2003

\section{Primary research}

Keywords: Lung cancer, PELP1, EGFR, TKI Drug resistance

Posted Date: July 30th, 2020

DOI: https://doi.org/10.21203/rs.3.rs-40995/v2

License: (c) (1) This work is licensed under a Creative Commons Attribution 4.0 International License. Read Full License 


\section{Abstract}

Background Lung cancer is one of the tumors with high morbidity and mortality among males and females worldwide. Proline-, glutamic acid-, and leucinerich protein 1(PELP1) is an estrogen receptor coactivator and functions as a scaffolding protein. Its oncogenic signaling was involved in the progression of several cancers. However, there is little known about the role of PELP1 in the lung cancer. The aim of this study is to explore the role of PELP1 in non-small cell lung cancer. Methods The expression of PELP1 in microarrays of lung cancer tissue and correlation with clinicopathological parameters were performed. RNA interference technology was used to downregulate PELP1 expression in A549 lung cancer cells and the tumor cell's ability of proliferation, migration, invasion was detected by Cell Count Kit-8 and trans-well essay. In addition, whole genome exon was analyzed by poly-A RNA-sequencing. Results Results showed that PELP1 is overexpressed in the lung cancer specimens and its expression correlate with histological type, smoking status, and EGFR status but no significant prognostic value. Knockdown of PELP1 inhibited cell proliferation, migration, invasion of lung cancer cells. Furthermore, silencing PELP1 in lung tumor cells promotes the sensitivity of tumor cells to the tyrosine kinase inhibitor Gefitinib. RNA-sequencing and Western-blotting found that 140 genes were up-regulated and 143 genes were down-regulated in PELP1 silenced lung adenocarcinoma cells, which mainly affected the mitogen-activated protein kinase (MAPK) signal pathway, EGFR tyrosine kinase inhibitor resistance, PPAR signaling pathway, Cytosolic DNA-sensing pathway, cytokine-cytokine receptor interaction, drug metabolism, cAMP signaling pathway, RIG-1-like receptor signaling pathway. Conclusions PELP1 plays a key role in non-small cell lung cancer proliferation, progression, and drug resistance. These results suggest that PELP1 could be a potential target for therapeutic intervention for lung cancer.

\section{Background}

The lung cancer tumor was one of the tumors with high morbidity and mortality among males and females worldwide which caused about 1.7 million deaths per year [1]. The incidence of lung cancer is rising rapidly. Among the top 10 global causes of death, it rose from ninth in 2000 to sixth in 2016 [2]. Adenocarcinoma and squamous cell carcinoma are the most common histopathologic types of non-small cell lung cancer (NSCLC) [3]. The epidemiologic investigation in recent years found that the incidence of lung adenocarcinoma exceeds the incidence of lung squamous cell carcinoma [4]. The evidence on shifting trends in lung cancer histological types was comprehensively reviewed in the United States surgeon general's 2014 report [5]. With the understanding of the molecular characteristic of lung cancer in latest two decades, molecular mechanisms such as EGFR-sensitive mutations have been discovered, and a new era of precise targeted treatment of lung cancer has been created [6,7]. Although EGFR-mutated lung cancers are very sensitive to tyrosine kinase inhibitors and lead to improvement in progression-free survival compared to standard first-line chemotherapy, drug resistance are inevitably [8,9]. Studies have found that a significant proportion of NSCLC specimens express estrogen receptor (ER) in tumor cell nuclei and in extranuclear sites. Furthermore, ERa in tumor nuclei was present in activated forms which residues at serines-118 and -167 were phosphorylated commonly modulated by growth factor receptor as well as steroid signaling [10].

Proline, glutamate, and leucine-rich protein 1 (PELP1) is a scaffolding protein that acts as a coregulator of multiple transcription factors and nuclear receptors, including ESR1, ESR2, AR, E2F and STAT3 [11,12]. PELP1 is expressed in a variety of tissues - the highest levels of expression found in the brain, testes, ovaries and uterus [13]. It is worth noting that the PELP1 protein has a histone binding domain that recognizes histone modifications and interacts with several chromatin modification complexes $[14,15]$. As a substrate for multiple kinases, PELP1 regulates its function in various complexes. In addition, PELP1 plays a vital role in a variety of pathways, including hormone signaling, cell cycle progression, ribosome biogenesis, and DNA damage responses [16,17,18]. PELP1 was recognized as proto-oncogene and its expression was dysregulated in several cancers including breast cancer, lung cancer, and ovary cancer $[19,20]$. It`s up-regulation or dislocation contributes to breast cancer cell proliferation, metastasis, and treatment resistance [21]. Furthermore, PELP1 was found been a prognostic indicator of decreased survival in patients with breast cancer [22]. Recent evidence suggests that PELP1 represents a new therapeutic target for many hormonal cancers [23].

Studies have found that there are increased levels of PELP1 transcript and protein in non-small cell lung cancer tumor tissues compared to adjacent normal lung tissues [24]. However, the exact function of PELP1 in lung cancer remains unknown. In this study, we detected the expression of PELP1 in 324 lung cancer tissues using immunohistochemistry and tissue microarray methods, and correlated with clinicopathological characteristics and survival status. We found that approximately $49 \%$ of lung cancer tissues expressed PELP1 diffusely. In addition, it has significant correlation with histological type, smoking status, and EGFR mutation. In vitro, silencing PELP1 gene can significantly inhibit the proliferation, migration, and metastasis of lung adenocarcinoma tumor cells. Furthermore, knockdown the PELP1 gene can increase the sensitivity of lung adenocarcinoma cells to the tyrosine kinase inhibitor (TKI) gefitinib. RNAsequencing and Western-blotting found that 140 genes were up-regulated and 143 genes were down-regulated in PELP1 silenced lung adenocarcinoma cells, which mainly affected the mitogen-activated protein kinase (MAPK) signal pathway, EGFR tyrosine kinase inhibitor resistance, PPAR signaling pathway, Cytosolic DNA-sensing pathway, cytokine-cytokine receptor interaction, drug metabolism, cAMP signaling pathway, and RIG-1-like receptor signaling pathway.

\section{Materials And Methods}

\section{Patients' data}

Archival paraffin embedded samples from lung cancer patients were retrieved from the author's hospital for tissue microarray (TMA) construction. All the specimens were routinely processed and stained with hematoxylin and eosin. The diagnosis was confirmed according to the tumor classification of world health organization. The clinical data of patients, including age, sex, TNM stage, and tumor size were retrieved from the medical records.

\section{Cell line and reagents}

Human lung cancer cells A549 were purchased from BOSTER biological technology co. Itd. (Wuhan, China). The cells were cultured in Dulbecco's modified Eagle medium (DMED) supplemented with $10 \% \mathrm{FBS}, 100 \mathrm{U} / \mathrm{mL}$ penicillin, and $100 \mathrm{mg} / \mathrm{mL}$ streptomycin. Cells were cultured under $37^{\circ} \mathrm{C}$ in $5 \% \mathrm{CO}_{2}$ and $95 \%$ 
humidified air. The antibodies of PELP1, GADPH, CyclinD1, MMP9, RET, p-MAPK were purchased from YRY bio-technology Ltd. (Guangzhou, China). EGF and Gefitinib were purchased from Zuoke bio-technology developing Ltd. (Guangzhou, China).

\section{Immunohistochemistry}

Sections from each TMA were stained with hematoxylin and eosin and reviewed to confirm the presence of representative tumors. 4-micron thick sections were processed to immunohistochemistry staining using an automated immunostaining instrument (Ventana Benchmark XT auto-stainer (Ventana Medical systems, Inc., Tucson, AZ, USA). After deparaffinized, rehydrated, and blocking the endogenous peroxidase activity, the sections were boiled in citrate buffer for 25 minutes. Then, incubating with PELP1 (1:500, polyclonal antibody, Bethyl Laboratories Inc., Montgomery, TX) for 25 min at $37^{\circ} \mathrm{C}$. After washing with PBS, the sections were treated with an ultraView Universal HRP multimer(Ventana Medical Systems), which contained a cocktail of HRP-labeled goat anti-rabbit and anti-mouse secondary antibodies, for 8 minutes at $37^{\circ} \mathrm{C}$. Then, visualized with 3, $3^{\prime}$-diaminobenzidine chromogen and counterstained with hematoxylin . The tumor cells in the TMA slides were assessed for the staining intensity and percentage of stained cells in the nucleus. The percentage of cells with moderate/strong nuclear staining was recorded. PELP1 staining was classified into low ( $<5 \%)$, focal (5-49\%), and diffuse (>=50\%).

\section{Western blotting analysis}

The treated cells were rinsed twice by PBS and lysed in RIPA buffer containing protease inhibitors (lysis buffer: protease inhibitor冈PMSF: phosphatase inhibitor were 1000:1:5:10), the supernatant were separated by a high speed centrifuge at $4^{\circ} \mathrm{C}$ and the protein concentrations were measured by a BCA protein assay reagent Kit (Bioworld). 30 ug proteins from each sample were separated by $10 \%$ sodium dodecyl sulfatepolyacrylamide gel electrophoresis and transferred onto polyvinylidene fluoride membranes (Thermo Fisher scientific, Waltham, MA, USA). Membranes were incubated with rabbit or mouse monoclonal antibody (1:1000 diluted) at $4^{\circ} \mathrm{C}$ overnight. After washed three times by PBST buffer (PBS added 0.05\% Tween 20), the membranes were incubated with horseradish peroxidase (HRP)-labeled goat anti rabbit and mouse secondary antibody (1:10000 diluted) for 1 hour at room temperature. The band was visualized by FCD ${ }^{T M} E C L$ Kit.

\section{RNA isolation and real-time PCR analysis}

Total RNA was isolated from the treated cell lines by Trizol reagents (Invitrogen, CA) according to the manufacturer spro $\rightarrow$ col. $2 \mu$ gof $\rightarrow$ talRNAomeachsamp $\leq$ was $\subset$ jected $\rightarrow$ cDNAsynthesis. $R T$-PCRanalyseswereperf or med $\in$ triplicatea or $d \in g \rightarrow t$ $s$ instruction (PrimeScript ${ }^{\mathrm{TM}} \mathrm{RT}$ Master Mix, TaKaRa, Japan) and GADPH was used as an internal control. The primer sequences were as follows: PELP1 forward: TCAGTAATGCACGTCTCAGTTC, PELP1 reverse: CTCCGAAGCCAAGACACACAG, GADPH forward: GGAGCGAGATCCCTCCAAAAT, GADPH reverse: GGCTGTTGTCATACTTCTCATGG.

\section{Knockdown of PELP1 by siRNA}

Small interfering RNA targeting PELP1 (siRNA1: GGCTTGTGGTTCTCTCAAA; siRNA2: CCAGGAGCTTGTTGAAGAA; siRNA3: CAAGGTGTATGCGATATTA) and negative control were purchased from RiboBIO Ltd. (Guangzhou, China) and transfected using riboFECT ${ }^{\mathrm{TM}} \mathrm{CP}$ transfection Kit (RiboBio Ltd., Guangzhou, China) according to the recommend protocol. SiRNAs were transected at a final concentration of 100nM. PELP1 knockdown was validated by western blot and RT-PCR 48 hours after the transfection.

\section{Proliferation assay}

Forty-eight hours after siRNA transfection, 200ul culture medium containing 1000 cells per well were added into 96 -well plates. After $24,48,72,96$ and 120 hours of culture, cell viability was detected respectively by Cell Counting Kit-8(CCK8). Three replicated experiment were performed.

\section{Trans-well invasion and migration assay}

The trans-well migration and invasion assays were performed in transwell chambers (Corning Inc., Corning, NY, USA). 200ul suspension containing $2 \times 10^{5}$ cells in serum-free DMEM were seeded into the upper chamber. The lower chambers contained $10 \%$ FBS medium as an attractant. After 24 hours incubation, the cells in the upper chamber were removed with cotton swabs and the migrated cells on the bottom of membranes were fixed in methanol and stained with $0.01 \%$ crystal violet dye solution. The number of migrating cells in five randomly selected fields was counted on $200 x$ microscopic field. The invasion assay was the same except that the upper chamber was treated by Matrigel (Clontech) before seeding. Three replicated experiments were performed.

\section{Colony formation assay}

$2 \mathrm{ml}$ complete culture medium containing 200 cells were added into 6-well plates. The cell culture was terminated once visible colonies were formed. Then cells were washed twice with $3 \mathrm{ml}$ PBS, fixed with methanol for 15 minutes and stained with $0.01 \%$ crystal violet dye solution for 20 minutes. Afterwards, the plates were washed with PBS and the number of colonies was counted and photographed after the plates are air-dry. The colony formation ratio was expressed as "number of colonies/200 ${ }^{*} 100(\%)$ ". Three replicated experiments were carried out.

\section{RNA sequencing and functional enrichment analysis}

Total RNA was isolated from cells using the Trizol (invitrogen) according to the manufacturer's protocol. RNA purity was assessed using the ND-1000 Nanodrop. Each RNA sample had an A260:A280 ratio above 1.8 and A260:A230 ratio above 2.0. RNA integrity was evaluated using the Agilent 2200 TapeStation (Agilent Technologies, USA) and each sample had the RIN above 7.0. Briefly, rRNAs were removed from Total RNA using EpicentreRibo-Zero rRNA

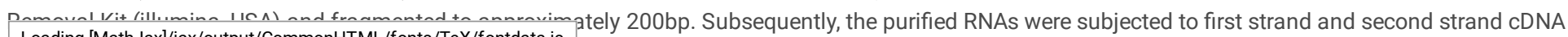


synthesis following by adaptor ligation and enrichment with a low-cycle according to instructions of NEBNext巴 Ultra ${ }^{\mathrm{T} M}$ RNA Library Prep Kit for Illumina (NEB, USA). The purified I library products were evaluated using the Agilent 2200 TapeStation and Qubit®2.0(Life Technologies, USA) and then diluted to 10 pM for cluster generation in situ on the pair-end flow cell followed by sequencing ( $2 \times 150$ bp) HiSeq 3000 . Clean reads were obtained by removing reads containing adapter, reads containing ploy- $\mathrm{N}$ and low quality reads from raw data. HISAT2 was used to align the clean reads to the mouse reference genome mm 10 with default parameters. HTSeq was subsequently employed to convert aligned short reads into read counts for each gene model. Differential expression was assessed by DEseq using read counts as input. The multiple testing correction method was enabled based on Benjamini-Hochberg procedure. Differentially expressed genes were chosen according to the criteria of fold change $>2$ and adjusted $p$-value $<0.05$. All the differentially expressed genes were used for heat map analysis and KEGG ontology enrichment analyses. For the KEGG enrichment analysis, a P-value $<0.05$ was used as the threshold to determine significant enrichment of the gene sets.

\section{Gefitinib sensitivity of A549 cells}

100ul cell suspensions were seeded into 96-well plates, after 3 hours, different concentrations of gefitinib $(2.5,5,10,20,30,40,60,80$ and 120 uM) were added to the culture medium. For negative control and blank group, the same amount of culture medium and PBS instead of gefitinib was added. After 72 hours incubation, 100ul CCK8 reagent was added into each well, and then incubated for 3 more hours. Absorbance (optical density, OD) at the 450 nm wavelength was detected. The cell survival rate was calculated according to the following formula: Cell survival rate\%= (drug group OD - blank group OD)/ (negative control group OD - blank OD) $\times 100 \%$.

\section{Statistical analysis}

The data were analyzed using the statistical software SPSS for window ver.19 (IBM Corporation, Armonk, NY). Chi-square analysis or Fisher exact test was used to test for the association of PELP1 expression with tumor grade and other clinical-pathologic parameters. Survival data was evaluated with KaplanMeier analysis with a log rank test. Statistical significance was established at $p<0.05$.

\section{Results}

\section{The expression of PELP1 in lung cancer and the relationship with clinicopathological features}

Totally, 324 lung cancers were included for this study of which containing 185 cases of adenocarcinoma, 98 cases of squamous carcinoma, 23 cases of other histopathologic subtypes. The average age was 64.4 years old. The mean size of tumor was $3.99 \mathrm{~cm}$ (details on the clinicopathological characteristics of the cohort were shown in table1).

PELP1 staining was located on nuclear, with no cytoplasmic or membranous staining in all tumor cells (figure 1). Overall, the positive expression rate of PELP1 in lung cancer was 49\%. There was significant difference of the PELP1 expression among adenocarcinoma and squamous carcinoma. High expression of PELP1 was significantly associated with squamous carcinoma, male, extensive smoker, and EGFR wild type. We found that the rate of PELP1 expression was three times more in EGFR wild type adenocarcinoma than the rate in EGFR mutant cases. No statistical correlations were found between PELP1 expression and age, tumor size, stage (table 2). We found no significant difference in OS and DFS between low and high PELP1 expression groups, although the disease-free survival of the high PELP1 express cases was longer than low express cases in EGFR mutant group (figure 2).

\section{Knockdown of PELP1 inhibited the proliferation, colony formation, migration, and invasion of A549 cells}

To explore the potential oncogenic function of PLEP1 in lung adenocarcinoma, we utilized siRNA to silence PELP1 expression in A549 cells and then the cell's ability of proliferation, migration, invasion, and colony formation were detected. Western-blot was used to verify the knockdown efficiency and the result showed siRNA2 is the best among the three sequences (figure3A). CCK8 assay found that the cell proliferation rate was significant decreased in PELP1 silenced A549 cells compared to the negative control cells $(P<0.01$, figure $3 C)$. In addition, the number of cell migration, invasion and colony formation was significantly reduced in PELP1 knockdown A549 cells compared with the negative control cells. These results suggested that silencing PELP1 inhibited the malignant behavior of $A 549$ cell in vitro $(P<0.01$, figure 3D, 3E, 3F).

\section{PELP1 knockdown downregulate MAPK signal pathway and upregulate the expression of RB1}

AKT signaling pathway and MAPK signaling pathway were one of the important mechanisms to promote proliferation in caner. RB protein can also influence cell's proliferation through E2F. We found that PELP1 knockdown can significantly reduce the proliferation in A549 cells. On this account, we detected the AKT and MAPK protein using western-blot method. The results showed that the phosphorylated MAPK protein was reduced in PELP1 knockdown cells compared with the negative control group. In addition, RB protein express was higher in PELP1 silenced group than negative control group. Phosphorylated AKT protein did not show evident difference between the three groups (figure 4A). Therefore, PELP1 may through MAPK signaling pathway to promote cancer cell proliferate.

\section{PELP1 Knockdown increased the sensitivity of A549 cells to the TKI drug Gefitinib}

By treating A549 lung cancer cells with gradient concentration of Gefitinib and then detecting the cell viability by CCK8 reagent, we found that comparing to the control group, the PELP1 silenced group need a lower concentration of Gefitinib to reach half-inhibited. In addition, we found that Gefitinib can reduce the expression of PELP1 protein in A549 cells, while EGF can increase the expression of PELP1 in tumor cells (Figure 5a, 5b).

The whole genome transcription profile of the PELP1 knockdown A549 cells.

Loading [MathJax]/jax/output/CommonHTML/fonts/TeX/fontdata.js 
To explore the potential mechanisms by which PELP1 promotes A549 cells invasion and metastasis and drug resistance, we sequenced the whole genome transcription profile of the PELP1 silenced A549 cells and compared with the controls. The results found that 140 genes were significantly upregulated. The most significantly different gene included CYP46A1, DHX58, MAPK4, ASGR2, MLIP, ZCWPW2, SLC22A1, LRRC71, SGCA, CFAP70. Gene ontology analysis showed they involve adrenergic receptor activity, oxidoreductase activity, monoamine transmembrane transporter activity, molybodopterin cofactor binding, intrinsic and integral component of membrane, and regulation of adenylate cyclase activity. KEGG pathways analysis found significantly upregulated pathway including EGFR tyrosine kinase inhibitor resistance, PPAR signaling pathway, Cytosolic DNA-sensing pathway, cytokine-cytokine receptor interaction, drug metabolism, cAMP signaling pathway, RIG-1-like receptor signaling pathway. In the meantime, 143 genes were found significantly downregulated and top of them including PRPH2, TAC4, CCDC8, SLC7A10, SPATA21, OXCT2, BSPRY, HIST1H2BN, ARMC3, LRRN4, RET. Gene ontology analysis showed those downregulated genes are related to SUMO polymer binding, secondary active transmembrane transporter activity, Ras guanyl-nucleotide exchange factor activity, death receptor activity, tumor necrosis factor-activated receptor activity, intrinsic and integral component of membrane. KEGG pathways analysis showed significantly downregulated pathway including complement and coagulation cascades, central carbon metabolism in cancer, retinol metabolism, cytokine-cytokine receptor interaction, thyroid cancer, and pentose phosphate pathway.

\section{Discussion}

PELP1 is a proto-oncogene which plays a key coregulatory protein role, providing distinct growth and survival advantages for cancer cells. In vitro, overexpression of PELP1 in fibroblasts and epithelial model cells results in cellular transformation. In vivo, deregulated PELP1 enhances the hormoneindependent growth of breast cancer cells in xenograft mouse models [24]. Furthermore, in one study [25], they generated an inducible, mammary glandspecific PELP1-expressing transgenic mouse and found more proliferation, extensive side branching, and precocious differentiation in PELP1-overexpressing mammary glands than in control glands and the mouse had hyperplasia and preneoplastic changes as early as 12 weeks. Studies using clinical breast cancer tissues found that the expression of PELP1 in node-positive and metastatic tumors increased comparing with node-negative specimens [24]. PELP1 protein expression is an independent prognostic indicator of shorter breast cancer specific survival and disease-free survival in breast cancer and its upregulate was positively correlated with markers of poor outcome [26]. In astrocytic brain tumors, increased expression of PELP1 has been associated with higher grade of tumors and worse prognosis [27]. Similar observations have been also found in prostate cancer and endometrioid carcinoma [28, 29]. However, one study found that PELP1 overexpression exists in epithelial cells of colorectal cancer and is an independent favorable prognostic factor [30]. We investigated the expression of PELP1 in 324 lung cancer archival samples and found that about $46.2 \%$ cases diffusely nuclear positive. Analysis found PELP1 expression in lung cancer correlates with histotype, EGFR status, smoking status, and sex. PELP1 expression in lung cancer tissues did not show association with overall survival and relapsed free survival. Although in EGFR positive group the relapse free survival is longer in PELP1 high cases than PELP1 low cases, but it did not show significant statistic difference.

Many studies have shown that PELP1 drives cell proliferation, invasion, and metastasis in hormonal tissues and cancers. In the mouse xenografts model, knockdown PELP1 of breast cancer cell MCF-7 can reduce the tumor volume and the Ki-67 proliferation index shows significantly decreased [31]. In the ovarian cancer cell line Ishikawa, silencing PELP1 significantly inhibited the migratory potential of tumor cells, observed by Boyden chamber assay. Moreover, knockdown PELP1 in Ishikawa cells resulted in significantly reduced cell motility and suppression of E2-induced cell migration effects. PELP1 downregulation altered the expression of matrix metalloproteinase (MMP)-2 and MMP-9 accounts for parts of its mechanism [32]. In ER-negative MDA-MB-231 cells, PELP1 knockdown can reduce the proliferation, migration and invasion in vitro. In vivo, using an MDA-MB-231 xenograft with PELP1 silenced showed a reduced tumor volume compared to control cells. This study also suggests that PELP1 expression may promote migration and invasion by regulating the epitheliamesenchymal transformation (EMT) gene. PELP1 knockdown leads to reduced expression of EMT genes, including MMP, ZEB1 / 2, SNAI1 and TWIST1 [33] PELP1 is physiologically related to the cyclin-switching protein retinoblastoma ( $p R b)$ in the nucleus, and these PELP1 - $\mathrm{pRb}$ interactions have been found to function in activating E2 target genes such as cyclin D1. It was identified Ser477 and Ser991 of PELP1 were cyclin-dependent kinase phosphorylation sites. PELP1 is recruited to pRB/E2F target genes and facilitates ER signaling cross talk with cell cycle machinery [34]. One study used MCF-7 breast cancer cells to generate a model system in which PELP1 was expressed only in the cytoplasm. They found that the PELP1 dislocation model cells were hypersensitive to estrogen but resistant to tamoxifen. In addition, the dislocation of PELP1 in cytoplasm increased PELP1 association with Src and MAPK activation [35]. Another study found that hepatocyte growth factor-regulated tyrosine kinase substrate sequester PELP1 in the cytoplasm leads to MAPK activation. This activation manner is dependent on EGFR, but independent of ER, Shc, Src [36]. Our study use siRNA silence PELP1 of lung adenocarcinoma A549 cell found that downregulate PELP1 significantly inhibit the ability of tumor cell proliferation, colony formation, migration and invasion. Western-blot results showed that knockdown PELP1 downregulate MAPK signal pathway and upregulate RB1 protein expression in A549 cells.

Estrogen receptors, including ER alpha and ER beta, have been found to be expressed in a significant portion of non-small cell lung cancer tissues. In addition, ER alpha in tumor nuclei was present in activated forms as the serines-118 and 167 residues were phosphorylated. EGFR and ER also cooperate in promoting early activation of MAKP pathway signal in NSCLC cells [37]. One study using in vivo labeling assays found that growth factors promote phosphorylation of PELP1 and this effect was through protein kinase K pathway [38]. Our result showed that growth factor signal can also upregulate the expression of PELP1 in vitro, the mechanisms should be further investigated. Studies have demonstrated that PELP1 direct physical interactions with both EGFR and ER. PELP1 may play a pivot role in the crosstalk between EGFR signal and ER pathway. The ability of PELP1 to interact with ER, chromatin components signaling enzymes (such as Src and PI3K), and growth factor signaling components (such as EGFR, STAT3, and HRS) suggests that PELP1 acts as a scaffold protein and plays a role in assembly a variety of signaling complexes and their crosstalk. Our study also showed silencing PELP1 expression in lung adenocarcinoma A549 cells can enhance the sensitivity of tumor cell to the EGFR inhibitor gefitinib.

To have a panoramic view of the mechanism by which PELP1 acts on tumor cells, we have performed a whole genome exon analysis to profile the PELP1 transcriptome by poly-A RNA sequencing. 140 genes were identified upregulated and 143 genes downregulated. Pathway analysis discovered that PELP1 modulates several pathways including the molecular mechanisms of cancer, EGFR tyrosine kinase inhibitor (TKI) resistance, PPAR signaling pathway, Loading [MathJax]/jax/output/CommonHTML/fonts/TeX/fontdata.js 
metabolic pathways, regulation of autophagy, RIG--like receptor signaling pathway, central carbon metabolism in cancer, and cytokine-cytokine receptor interaction. Some of those pathways have been verified experimentally by our or others studies. Most of it needs to be further confirmed and investigated. Among those significant differential express genes, RET was recognized oncogene, MAPK4 were components of MAPK pathway, SPEF1 and SPATA2 involved in spermatogenesis may partly account for the PELP1 that was found to be a biological marker of sperm morphology abnormalities [39], NRG2 was participated in the EGFR TKI drug resistance.

\section{Conclusions}

The main findings of this study were: (1) PELP1 was highly expressed in non-small lung cancer and its expression has been correlated with histotype, EGFR status, smoking status, and sex. PELP1 was not found to associate with survival of patients with lung cancer. (2). Knockdown of the expression of PELP1 significantly inhibited the proliferation and invasion of the lung adenocarcinoma cells. (3) Silencing PELP1 downregulate MAPK signal pathway and upregulate the expression of RB1. (4) PELP1 knockdown increased the sensitivity of lung adenocarcinoma cell to the TKI drug Gefitinib. (5) Our study revealed the whole genome transcription profile of the PELP1 acting on lung cancer cells. Generally, this work demonstrated that PELP1 plays important role in lung cancer cell proliferation, invasion, metastasis, and drug resistance which suggested the possibility of PELP1 as a potential therapeutic target.

\section{References}

[1]. Rebecca L Siegel, Kimberly D Miller, Ahmedin Jemal. Cancer statistics, 2019. CA Cancer J Clin. 2019; 69:7-34.

[2]. World Health Organization. Global health estimates 2016: Deaths by Cause, Age, Sex, by country and by region, 2000-2016. Geneva, 2018.

[3]. L. Esposito, D. Conti, R. Ailavajhala, N. Khalil, et al. Lung cancer: are we up to the challenge? Curr. Genomics. 2010; 11:513-518.

[4]. travis WD, Brambilla E, Noguchi M, et al. international association for the study of lung cancer/American thoracic society/European respiratory society international multidisciplinary classification of lung adenocarcinoma. J Thorac Oncol. 2011; 6:244-85.

[5]. United states public health service office of the surgeon general and national center for chronic disease prevention and health promotion office on smoking and health the health consequences of smoking- 50 years of progress: a report of the surgeon general. U.S. department of health and human services, centers for disease control and prevention, national center for chronic disease prevention and health promotion, office on smoking and health 2014.

[6]. Lynch TJ, Bell DW, Sordella R, et al. Activating mutations in the epidermal growth factor receptor underlying responsiveness of non-small-cell lung cancer to gefitinib. New England journal of medicine. 2004;350(21):2129-39.

[7]. Paez JG, Janner PA, Lee JC, et al. EGFR mutations in lung cancer: correlation with clinical response to gefitinib therapy. Science. 2004;304(5676):14971500 .

[8]. Maemondo M, Inoue A, Kobayashi K, et al. Gefitinib or chemotherapy for non-small -cell lung cancer with mutated EGFR. New England Journal of Medicine. 2010;362(25):2380-2388.

[9]. Mok TS, Wu YL, Thongprasert S, et al. Gefitinib or carboplatin-paclitaxel in pulmonary adenocarcinoma. New England Journal of Medicine. 2009;361(10):947-957.

[10]. Diana C.M, Hsiao-Wang Chen, Michael C.F, et al. Estrogen receptor signaling pathways in human non-small cell lung cancer. Steroids.2007;72(2):135-43.

[11]. Girard BJ, Daniel AR, Lange CA, et al. PELP1: a review of PELP1 interactions, signaling, and biology. Mol Cell Endocrinol. $2014 ; 382: 642-651$.

[12]. Brann DW, Zhang QG, Wang RM, Mahesh VB, Vadlamudi RK. PELP1-a novel estrogen receptor interacting protein. Mol Cell Endocrinol. 2008 ; $290: 2-7$.

[13]. R K Vadlamudi, R A Wang, A Mazumdar, et al. Molecular cloning and characterization of PELP1, a novel human coregulator of estrogen receptor alpha. J Biol Chem. 2001; 276:38272-9.

[14]. Monica Mann, Valerie Cortez, Ratna Vadlamudi. PELP1 oncogenic functions involve CARM1 regulation. Carcinogenesis. 2013;34(7):1468-1475.

[15]. Young Bong Choi, Jin Kyoung Ko, Jaekyoon Shin. The transcriptional corepressor, PELP1, Recruits HDAC2 and masks histones using two separate domains. The journal of biological chemistry. 2004:279(49):50930-41.

[16]. Castle CD, Cassimere EK, Denicourt C. LAS1L interacts with the mammalian Rix1 complex to regulate ribosome biogenesis. Mol Biol Cell. 2012; 23:716728.

[17]. Finkbeiner E, Haindl M, Muller S. The SUMO system controls nucleolar partitioning of a novel mammalian ribosome biogenesis complex. EMBO J. 2011; 30:1067-1078.

[18]. Gonugunta VK, Nair BC, Rajhans R, Sareddy GR, Nair SS, Vadlamudi RK. Regulation of rDNA transcription by proto-oncogene PELP1. PLoS One. 2011; 6: e21095.

[19]. Xingen Wang, Julia Y.S Tsang, Michelle A Lee, et al. the clinical value of pelp1 for breast cancer: a comparison with multiple cancers and analysis in Loading [MathJax]/jax/output/CommonHTML/fonts/TeX/fontdata.js -717. 
[20]. Dimple C, Nair SS, Rajhans R, Pitcheswara PR, Liu J, Balasenthil S, Le XF, Burow ME, Auersperg N, Tekmal RR, Broaddus RR, Vadlamudi RK. Role of PELP1/MNAR signaling in ovarian tumorigenesis. Cancer Res. 2008; 68:4902-4909.

[21]. Cortez V, Samayoa C, Zamora A, Martinez L, Tekmal RR, Vadlamudi RK. PELP1 overexpression in the mouse mammary gland results in the development of hyperplasia and carcinoma. Cancer Res. 2014; 74:7395-7405.

[22]. Habashy HO, Powe DG, Rakha EA, Ball G, Macmillan RD, Green AR, Ellis IO. The prognostic significance of PELP1 expression in invasive breast cancer with emphasis on the ER-positive luminal-like subtype. Breast Cancer Res Treat. 2010; 120:603-612.

[23]. Chakravarty D, Roy SS, Babu CR, Dandamudi R, Curiel TJ, Vivas-Mejia P, Lopez-Berestein G, Sood AK, Vadlamudi RK. Therapeutic Targeting of PELP1 Prevents Ovarian Cancer Growth and Metastasis. Clin Cancer Res. 2011; 17:2250-2259.

[24]. Rajhans R, Nair S, Holden AH, et al. oncogenic potential of the nuclear receptor coregulator proline-, glutamic acid-, leucine- rich protein 1/ modulator of the nongenomic actions of the estrogen receptor. Cancer Res. 2007 Jun 1;67(11):5505-12.

[25]. Valerie Cortez, Cathy Samayoa, Andrea Zamora, et al. PELP1 overexpression in the mouse mammary gland results in the development of hyperplasia and carcinoma. Cancer Res. 2014,15;74(24):7395-405.

[26]. Habashy HO, Powe DG, Rakha EA, et al. The prognostic significance of PELP1 expression in invasive breast cancer with emphasis on the ER-positive luminal-like subtype. Breast Cancer Res Treat. 2010,120(3):603-12.

[27]. Kefalopoulou Z, Tzelepi V, Zolota V, et al. Prognostic value of novel biomarkers in astrocytic brain tumors: nuclear receptor co-regulators AIB1, TIF2, and PELP1 are associated with high tumor grade and worse patient prognosis. J Neurooncol. 2012 Jan;106(1):23-31.

[28]. Nair SS, Guo Z, Mueller JM, et al. Proline-, glutamic acid-, and leucine-rich protein-1/modulator of nongenomic activity of estrogen receptor enhances androgen receptor functions through LIM-only coactivator, four-and-a-half LIM-only protein 2. Mol Endocrinol. 2007 Mar;21(3):613-24.

[29]. Aust S, Horak P, Pils D, et al. The prognostic value of estrogen receptor beta and proline-, glutamic acid- and leucine-rich protein 1 (PELP1) expression in ovarian cancer. BMC Cancer. 2013, (14)13:115.

[30]. Petros Grivas, Vassiliki Tzelepi, Georgia Sotiropoulou-Bonikou, et al. Expression of ERalpha, ERbeta and co-regulator PELP1/MNAR in colorectal cancer: prognostic significance and clinicopathologic correlations. Cell Oncol. 2009;31(3):235-47.

[31] Rajib Rajhans, Sujit Nair, Alan H.Holden, et al. oncogenic potential of the nuclear receptor coregulator proline- glutamic acid-, leucine-rich protein $1 /$ modulator of the nongenomic actions of the estrogen receptor. Cancer research. 2007;67(11):5505-5512.

[32] Jing wan, Xiaomao Li. Pelp1/mnar suppression inhibits proliferation and metastasis of endometrial carcinoma cells. Oncology reports. 2012;28: 20352042.

[33]. Roy, S., Chakravarty, D., Cortez, V., De Mukhopadhyay, K., Bandyopadhyay, A., Ahn, J.M., Raj, G.V., Tekmal, R.R., Sun, L., Vadlamudi, R.K., 2012. Significance of PELP1 in ER-negative breast cancer metastasis. Mol. Cancer Res. 10, 25-33.

[34]. Nair BC, Nair SS, Chakravarty D, et al. Cyclin-dependent kinase-mediated phosphorylation plays a critical role in the oncogenic functions of PELP1. Cancer Res. 2010 Sep 15;70(18):7166-75.

[35]. Ratna Vadlamudi, Bramanandam Manavathi, Seetharaman Balasenthil, et al. Functional implications of altered subcellular localization of PELP1 in breast cancer cells. Cancer Res. 2005; 65(17):7724-32.

[36]. Suresh K Rayala, Petra den Hollander, Seetharaman Balasenthil, et al. Hepatocyte growth factor-regulated tyrosine kinase substrate (HRS) interacts with PELP1 and activates MAPK. J Biol Chem. 2006; 281(7):4395-403.

[37] Diana C.Marquez-Garban, Hsiao-Wang Chen, Michael C.fishbein, et al. estrogen receptor signaling pathways in human non-small cell lung cancer. Steroids.2007;72:135-143.

[38] Jatin K.Nagpal, Sujit Nair, Dimple Chakravarty, et al. growth factor regulation of estrogen receptor coregulator pelp1 functions via protein kinase a pathway. Mol cancer Res. 2008;6(5):851-861.

[39]. Yunxiang Zhao, Ning Gao, Xinyun Li, et al. identifying candidate genes associated with sperm morphology abnormalities using weighted single-step GWAS in a Duroc boar population. Theriogenology. 2020; 141:9-15.

\section{Abbreviations}

PELP1: proline, glutamate and leucine-rich protein 1;

NSCLC: non-small cell lung cancer; 
TMA: tissue microarray;

TKI: tyrosine kinase inhibitor;

DMED: Dulbecco`s modified eagle medium;

CCK8: Cell Counting Kit-8;

MAPK: mitogen-activated protein kinase

AKT: the serine-threonine kinase;

KEGG: Kyoto encyclopedia of genes and genomes;

MMP: matrix metalloproteinase;

EMT: epithelia mesenchymal transformation;

NRG2: neuregulin 2;

SPEF1: sperm flagellar1;

SPATA21: spermatogenesis associated 21;

OD: optical density;

\section{Declarations}

\section{Ethics approval and consent to participate}

Ethical approval was obtained from the competent institutional review boards of the Shenzhen hospital and Southern medical university.

\section{Consent for publication}

All authors read and approved the final manuscript.

\section{Availability of data and materials}

Not applicable.

\section{Competing interests}

The authors declare that they have no competing interests.

\section{Funding}

The study was supported by grants from "San-ming" project of Medicine in Shenzhen (NO. SZSM20182088, Weihua Yin)

\section{Authors' contributions}

All authors read and approved the final manuscript. LL, YWH and WXG contributed to concept and design this research. WXG performed the experiment and wrote the manuscript. LL and YWH revised the manuscript.

\section{Acknowledgements}

The authors are grateful for the technical helps of the other members of Li Liang Lab.

\section{Tables}

Please see the supplementary files section to view the tables.

\section{Figures}


Figure 1.
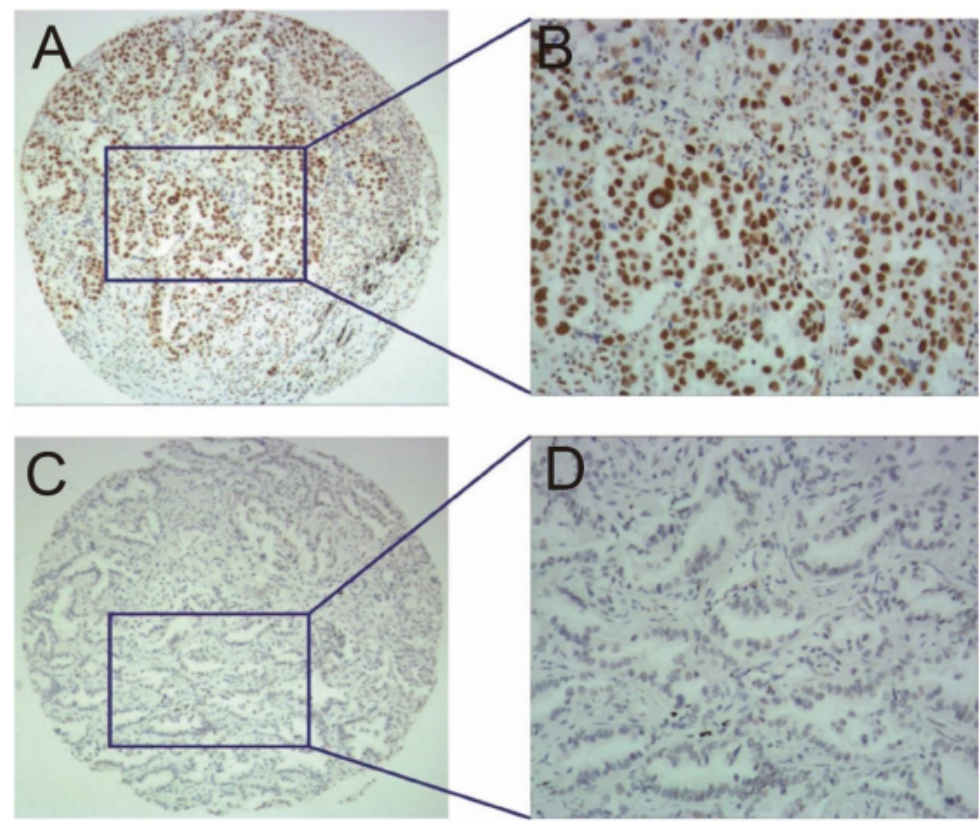

\section{Figure 1}

representative immunohistochemical staining of proline, glutamic acid, and leucine-rich protein 1 on lung cancers (A, B cancer cells diffuse nuclear positive; C, D negative staining)

Figure 2 .
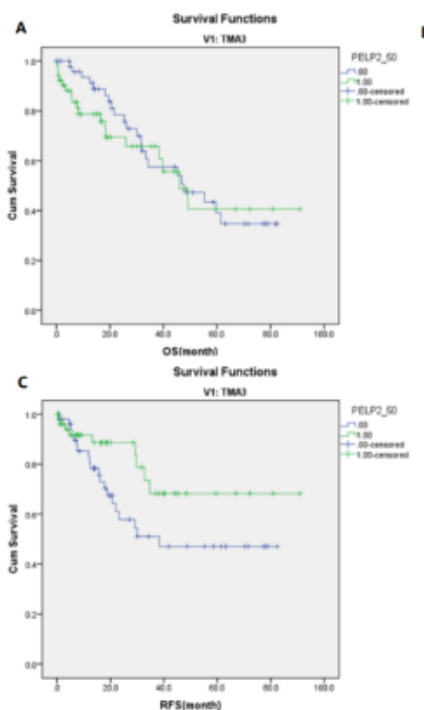
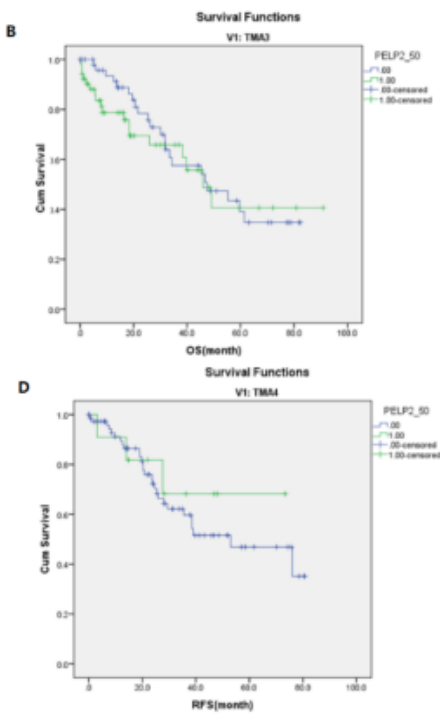

\begin{tabular}{|l|l|l|}
\hline & Log-rank & P-value \\
\hline EGFR+OS & .290 & .590 \\
\hline EGFR+RFS & 3.453 & .063 \\
\hline EGFR-OS & .028 & .867 \\
\hline
\end{tabular}

\begin{tabular}{l|l|l}
\hline EGFR-RFS & .304 & .867 \\
\hline
\end{tabular}

\section{Figure 2}

Kaplan-Meier analysis of proline, glutamic acid, and leucine-rich protein 1 expression for disease free survival and lung cancer specific survival in EGFR+ lung cancer cases $(A, C)$ and EGFR- lung cancer cases $(B, D)$. 

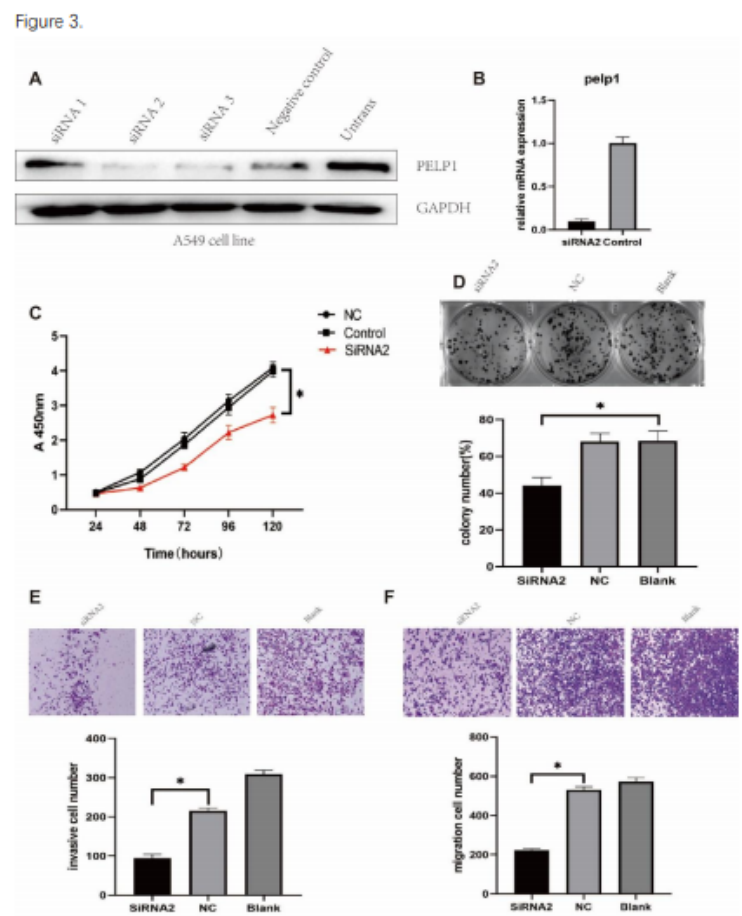

\section{Figure 3}

knockdown PELP1 inhibit lung cancer A549 cell proliferation, migration, invasion and colony formation. (A, B: the efficiency of PELP1 siRNA were detected by Western blotting and real-time PCR, siRNA2 was chosen for further investigation; C: cell proliferation potential of PELP1 silenced A549 cells and control were measured in vitro by CCK8 essay; D: PELP1 silencing inhibited the ability of colony formation of A549 cells significantly; E, F: the potential of migration and invasion were decreased significantly in A549 cell with PELP1 knockdown compared with control. *, $p<0.05, t$ test).

Figure 4 .

A

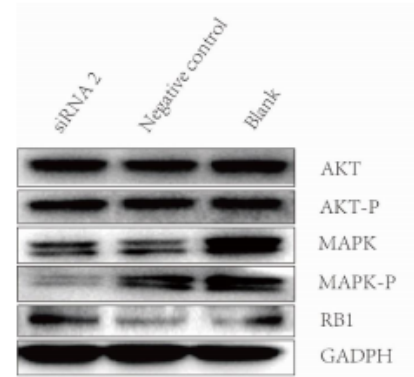

A549 cell line

B

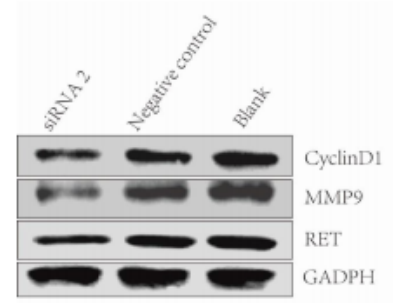

A549 cell line

\section{Figure 4}

4A. Western blotting results showed that phosphorylated MAPK was down-regulated and RB1 protein was up-regulated in PELP1 silenced A549 cells compare to control. 4B. Western blotting showed cyclinD1, MMP9 and RET protein down expressed in PELP1 silenced A549 cells compare to control. 

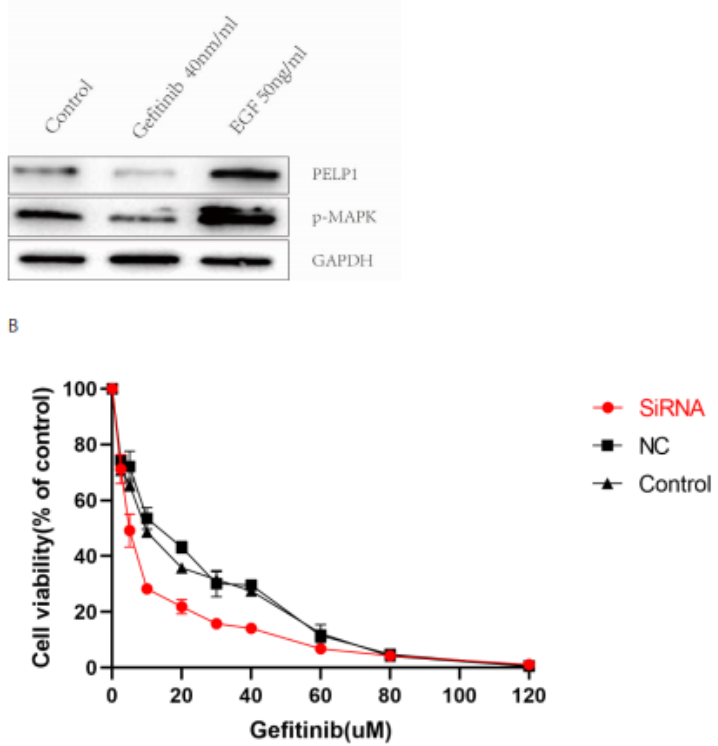

Figure 5

5A. PELP1 expression and phosphorylated MAPK were down-regulated in A549 cells after treated with 40nmol/ml gefitinib 48 hours late, and were upregulated when treated with $50 \mathrm{ng} / \mathrm{ml}$ EGF detected by Western blotting. 5B. A549 tumor cells were treated with gefitinib at a gradient concentration, and the sensitivity to the drug was significantly increased in the PELP1 knockdown group.

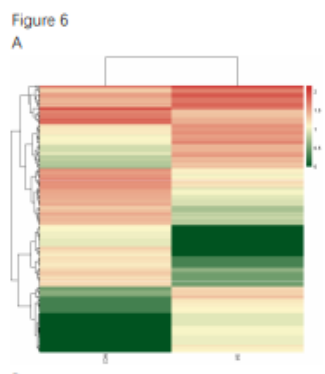

B
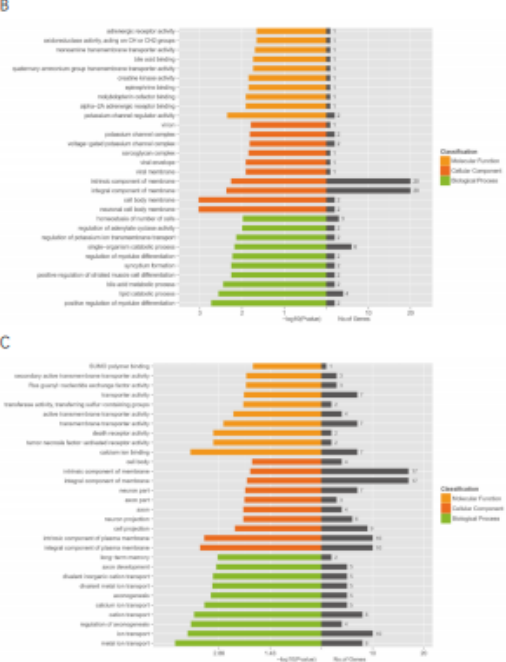

Figure 6

Analysis of whole transcriptional changes modulated by PELP1 in lung cancer cells. RNA isolated from PELP1 silenced A549 cells or control cells was sequenced by poly-A RNA sequencing methods. 6A: heatmap of differentially expressed genes between PELP1 knockdown A549 cells and control cells was shown. 6B, 6C: Gene ontology analysis of up and down differentially expressed genes in terms of biological processes, molecular functions and cellular component was performed with KOBAS3.0 software. 


\section{Supplementary Files}

This is a list of supplementary files associated with this preprint. Click to download.

- tablesandfigures.pdf 